\title{
Action goals influence action-specific perception
}

\author{
RouWen CAÑal-Bruland \\ VU University Amsterdam, Amsterdam, The Netherlands \\ AND \\ JOHN VAN DER KAMP \\ VU University Amsterdam, Amsterdam, The Netherlands \\ and University of Hong Kong, Hong Kong, China
}

\begin{abstract}
We examined the processes that mediate the emergence of action-specific influences on perception that have recently been reported for baseball batting and golf putting (Witt, Linkenauger, Bakdash, \& Proffitt, 2008; Witt \& Proffitt, 2005). To this end, we used a Schokokusswurfmaschine: Children threw a ball at a target, which, if hit successfully, launched a ball that the children then had to catch. In two experiments, children performed either a throwing-and-catching task or a throwing-only task, in which no ball was launched. After each task, the size of the target or of the ball was estimated. Results indicate that action-specific influences on perceived size occur for objects that are related to the end goal of the action, but not for objects that are related to intermediate action goals. These results suggest that action-specific influences on perception are contingent upon the primary action goals to be achieved.
\end{abstract}

Recently, Witt and colleagues (Witt, Linkenauger, Bakdash, \& Proffitt, 2008; Witt \& Proffitt, 2005) provided empirical evidence in support of frequently reported anecdotes that baseball batters see the ball as being bigger than usual when hitting well, and golfers judge the hole to be bigger on successful days. More specifically, Witt and Proffitt (2005) presented softball players who had just finished their game with a poster showing eight circles and asked them to choose the circle that they thought best matched the size of a softball. They found that size of chosen circles was positively correlated with the players' batting average. That is, players with higher batting scores tended to select larger circles, whereas their less successful counterparts judged the ball to be smaller. Witt et al. (2008) reported a similar finding among golf players, indicating that golfers who putted well estimated the hole to be bigger than did golfers who putted less successfully. Witt and Proffitt concluded that perception of the environment is not entirely determined by optical information, but is also influenced by nonvisual performance-related factors (e.g., Proffitt, 2006). This is referred to as actionspecific influence on perception (Witt \& Proffitt, 2008).

The processes that mediate the emergence of actionspecific influence on perception need further clarification. We propose that, in one such process, objects that are intimately linked to the goal of an action get perceptually accentuated. This proposal is derived from the functional perception theory, which holds that the intention (or motivation) to achieve a goal affects the perception of the objects relevant to the attainment of that goal (Bruner, 1957;
Bruner \& Postman, 1949). Bruner and colleagues reasoned that, when a person is motivated to act on a goal object, the relevant object becomes perceptually accentuated (e.g., is perceived as being bigger), so that it stands out among other environmental stimuli. Although the early evidence for the functional perception theory has been discredited by contradictory findings and methodological weaknesses (e.g., Klein, Schlesinger, \& Meister, 1951), recent empirical and theoretical work on embodied cognition is consistent with one of its main notions - namely, that visual perception is intrinsically linked with action intentions (e.g., Bekkering \& Neggers, 2002; Proffitt, 2006). For example, Veltkamp, Aarts, and Custers (2008) have recently shown that observers who are deprived of fluid and are (subconsciously) primed to drink perceive a glass of water to be bigger than it actually is. However, there was no such effect when participants were not primed to drink. Veltkamp et al. concluded that the intention to reach a goal creates a state of readiness for action that "impinges on basic perceptual processes" (p. 723). It follows that action-specific influences on perception are contingent upon the action goals to be achieved. In softball batting, the ball is the object that is intrinsically related to the action goal, and, in line with predictions from the perceptual accentuation hypothesis, its perception is affected (Witt \& Proffitt, 2005).

In the present study, we evaluated the role of perceptual accentuation in action-specific perception by using a sequential task, in which the primary or end goal of the action could be reached only through an intermedi-

R. Cañal-Bruland, r.bruland@fbw.vu.nl 
ate goal. We used a Schokokusswurfmaschine (SWM; see Figure 1), which is a traditional German children's game used at birthday and summer parties. Children throw a ball toward a target, which, if it is hit successfully, launches a Schokokuss (a chocolate-marshmallow) that the children then try to catch with their hands (or, for even more fun, with their mouths). In this sequential action, the throwing action is subordinate to the catching action. The target to which the ball is thrown, therefore, is an intermediate goal object; the launched object is the end goal object. It is to this end goal object that children's motivation is primarily directed.

The perceptual accentuation hypothesis predicts strong action-specific influences for the end goal object, but only weak influences, if any, for the intermediate goal object. Note that the perceptual accentuation hypothesis further predicts that a shift in the children's primary motivation toward achieving the action goal related to the intermediate object (i.e., to hit the target rather than to catch the object) should result in clear action-specific influence on perception of the formerly intermediate object, even if action production and accuracy are similar.

In two separate experiments, we tested these predictions emanating from the perceptual accentuation hypothesis. First, we compared children's perception of the size of the to-be-hit target after they performed a throwingonly task with that after they performed a throwing-andcatching task (Experiment 1). Second, we examined children's judgment of the size of the to-be-caught object after they performed the sequential throwing-and-catching task (Experiment 2).

\section{EXPERIMENT 1}

In Experiment 1, we examined whether the emergence of an action-specific influence on the perception of the size of the target toward which the ball is thrown depends on the children's motivation to achieve distinct action goals. Children performed a throwing-only task and a throwing-and-catching task and were asked to estimate the size of the target immediately after finishing each task. In the throwing-only task, no object was launched. Because there was no object to be caught, the children were motivated to hit the target, the target was, therefore, the end goal object. The perceptual accentuation hypothesis would predict action-specific effects on the perception of the target's size. By contrast, for the sequential throwingand-catching task, in which the target was the intermediate goal object and the launched ball was the end goal object, the perceptual accentuation hypothesis would predict no, or only very weak, action-specific influence on target-size perception.

\section{Method}

Participants. Twenty-three children $(13$ girls, mean age $=$ 5.5 years, $S D=0.5$ ) from a kindergarten in Senden, Germany, participated in the experiment. Six children threw left-handed. Parents provided prior written consent, and the experiment was conducted in accordance with the local institution's ethical guidelines.

Apparatus and Stimulus. An SWM was used (Figure 1). Children threw a softball $(9.5 \mathrm{~cm}$ in diameter) toward the SWM's circleshaped target $(20 \mathrm{~cm}$ in diameter, with its center $1.30 \mathrm{~m}$ above the ground). We used the SWM in two separate conditions: a throwingonly condition and a throwing-and-catching condition. The children

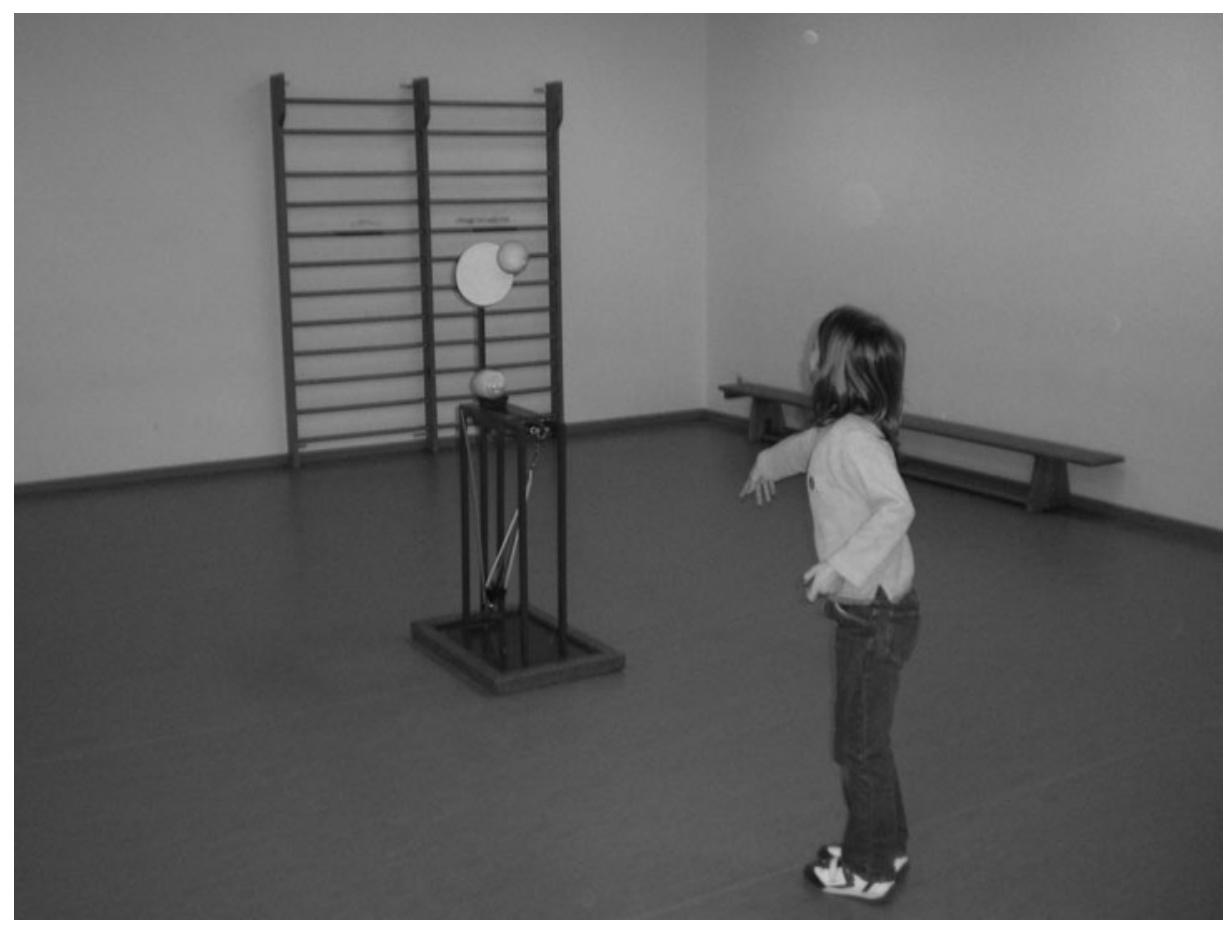

Figure 1. The Schokokusswurfmaschine. A child performs the throwing-and-catching task of Experiment 1. 
stood behind a white line at a distance of $1.30 \mathrm{~m}$ from the SWM, resulting in an effective target distance of approximately $1.85 \mathrm{~m}$. In the case of a hit, a mechanical lever connected to the target launched a second softball with a smile painted on it, which the children then tried to catch with their hands (in the throwing-and-catching task only). A pilot study had shown that, for children of this age, these distances resulted in the number of hits being normally distributed and resulted in the second ball approaching the children at chest height.

For the perception tests, we used nine black circles, each printed on a separate A3-size sheet of white paper. The circles measured between $0.18 \mathrm{~m}$ and $0.22 \mathrm{~m}$ in diameter, in increments of $0.5 \mathrm{~cm}$.

Procedure. Children were tested individually. They were familiar with the game and were keen to participate. For the throwing-only condition, the children were told to throw a ball and hit the target. A second to-be-launched ball was not present. For the throwing-andcatching condition, the children were told that they had to catch the ball with the smile on it after it was launched, and that they could launch the ball by throwing the other ball at the circle-shaped target. The experimenter demonstrated how the SWM launched the second ball by pushing against the target with his hand. The children were encouraged to catch this ball. Half of the children first performed the throwing-only task, whereas the other half first performed the throwing-and-catching condition. The children performed 15 trials in both conditions.

After each of the two conditions, the children performed a perception test. To this end, they were taken to the other end of the gym, where the sheets with the circles on them were arranged on the floor in a random fashion (i.e., the positions of the sheets were randomly distributed and changed after each test). In both tests, the children were instructed to look carefully and pick the circle they thought best corresponded to the size of the SWM's circle-shaped target. The children were allowed to look at the SWM while performing the perception tests. All children received a chocolate-marshmallow for their participation.

Data analysis. The number of balls that hit the target was taken as the main measure of performance. For the throwing-and-catching task, we also counted the number of balls that were caught. The diameter of the circle that the child chose was taken as the measure for size perception of the circle-shaped target. Spearman rho correlations were performed to examine the strength of the relation between the performance and size-perception measures for both conditions separately. In addition, paired $t$ tests were used to compare performance and size perception in the two conditions.

\section{Results and Discussion}

The results revealed a significant correlation between performance and size perception of the target in the throwing-only condition $(r=.402, p<.05)$ (see Figure 2A). Thus, in the throwing-only tasks, where the circle-shaped target was the primary action-related object, children who hit the target more often perceived the target to be bigger than did children who hit the target less successfully. This provides clear support for the perceptual accentuation hypothesis (see Veltkamp et al., 2008) and is in accordance with previous findings by Witt and colleagues (Witt et al., 2008; Witt \& Proffitt, 2005; see also Wesp, Cichello, Gracia, \& Davis, 2004).

By contrast, there was no significant relationship between performance (i.e., the number of balls that hit the target) and size perception when children were instructed to catch the ball that was launched when they hit the target $(r=-.033, p>.1)$ (see Figure 2B). ${ }^{1}$ When the children's motivation was primarily directed toward catching the launched ball — making the circle-shaped target the inter-
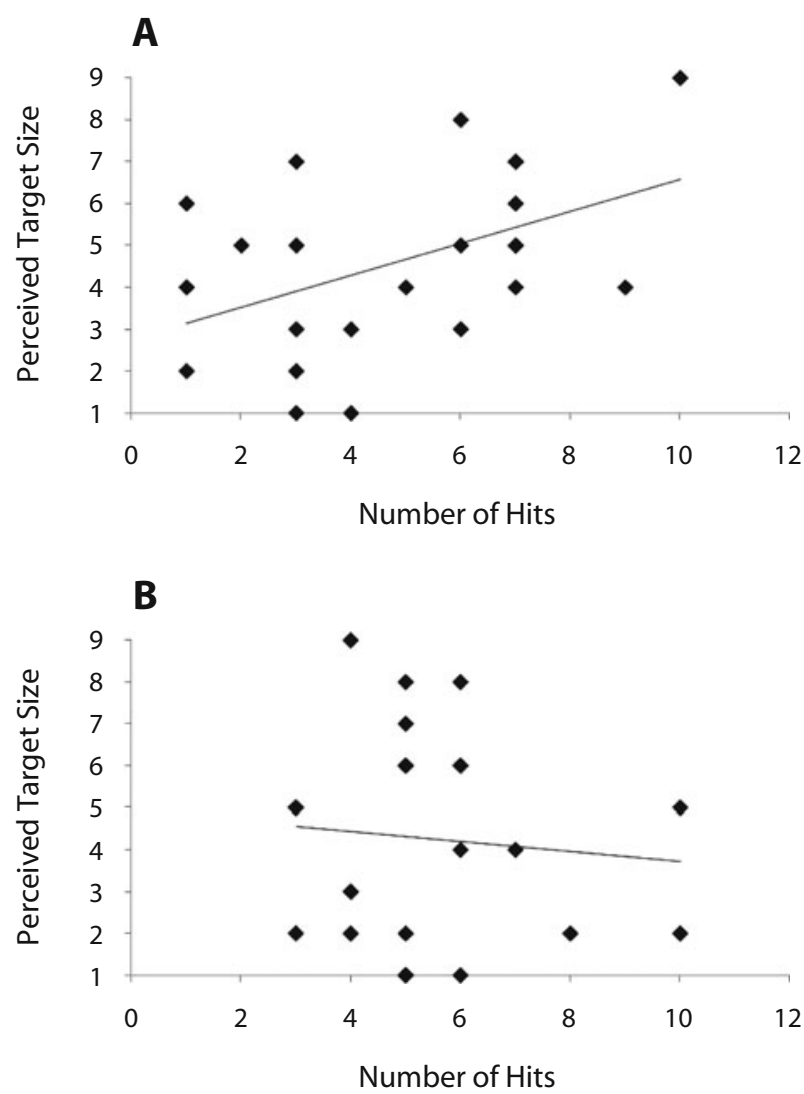

Figure 2. The relation between the number of hits and perceived target size, ranging systematically from 1 (smallest) to 9 (biggest) for the throwing-only condition (panel $A$ ) and for the throwingand-catching condition (panel B) in Experiment 1. Each dot represents 1 or more participants.

mediate goal object - the action-specific effect that was apparent in the throwing-only condition vanished. However, performance, as indicated by the number of hits, did not differ between the throwing-only and throwing-andcatching conditions $[t(22)=1.52, p>.1]$, nor did the size judgments significantly differ between the two conditions $[t(22)=0.77, p>.1]$ (see Table 1). Hence, our results provide evidence that action-specific perception is limited to the end goal object of an action and does not encompass intermediate goal objects.

By using generalized estimating equations (GEE; see, e.g., Liang \& Zeger, 1993), we further substantiated these findings by testing whether the slopes of the two regression lines for the correlation between the number of hits and size perception in the throwing-only and the throwing-and-catching conditions significantly differed. This regression analysis considers the measurements within subjects as repeated measures and accounts for this dependency. We computed the following regression equation: size perception $=\beta_{0}+\beta_{1} *$ hits $+\beta_{2} *$ condition $+\beta_{3} *$ hits $*$ condition. The regression analysis estimated the regression coefficients as $\beta_{0}=19.7, \beta_{1}=$ $-0.13, \beta_{2}=-0.90$, and $\beta_{3}=0.20$. The coefficient of the interaction term (i.e., $\left.\beta_{3}\right)$ was significant $(p=.03)$, 
Table 1

Mean Number and Standard Deviations of Target Hits and Caught Balls and the Perceived Target and Ball Size (Mean Diameters and Standard Deviations, in Centimeters) for the Throwing-Only and Throwing-and-Catching Tasks in Experiments 1 and 2

\begin{tabular}{|c|c|c|c|c|c|c|}
\hline & \multicolumn{4}{|c|}{ Experiment 1} & \multirow{2}{*}{\multicolumn{2}{|c|}{$\begin{array}{c}\text { Experiment } 2 \\
\text { Throwing- } \\
\text { and- } \\
\text { Catching }\end{array}$}} \\
\hline & \multicolumn{2}{|c|}{$\begin{array}{c}\text { Throwing- } \\
\text { Only }\end{array}$} & \multicolumn{2}{|c|}{$\begin{array}{l}\text { Throwing- } \\
\text { and- } \\
\text { Catching }\end{array}$} & & \\
\hline & $M$ & $S D$ & $M$ & $S D$ & $M$ & $S D$ \\
\hline Hits & 4.9 & 2.6 & 5.4 & 1.9 & 4.8 & 2.8 \\
\hline Caught balls & - & - & 2.3 & 2.6 & 1.9 & 2.7 \\
\hline Target size & 19.7 & 1.1 & 19.6 & 1.2 & - & - \\
\hline Ball size & - & - & - & - & 6.7 & 0.6 \\
\hline
\end{tabular}

indicating that the slopes of the two regression lines for the two conditions differed significantly. This finding is in accordance with the perceptual accentuation hypothesis, which claims that objects are only perceptually accentuated when they coincide with the observer's primary motivation for action. We further showed that performance efficacy mediates the degree to which the goal objects are accentuated (i.e., are perceived as being bigger).

\section{EXPERIMENT 2}

Even though the findings of Experiment 1 suggest that the perceptual accentuation hypothesis may theoretically explain the present findings, further evidence is needed in order to rule out the possibility that the disappearance of an action-specific influence on perception is not due to incomplete forward planning of the sequential task in young children, to task complexity, or, simply, to forgetting. In Experiment 2, we therefore examined whetherin the more complex sequential throwing-and-catching task - the action-specific perceptual accentuation indeed shifts toward the end goal-object (i.e., toward the launched ball).

\section{Method}

Participants. Twenty-six children participated in Experiment 2 (13 girls, mean age $=5.6$ years, $S D=0.5), 19$ of whom had also participated in Experiment 1, just 3 weeks before. Parents provided prior written consent, and the experiment was conducted in accordance with the local institution's ethical guidelines.

Apparatus and Stimulus. The SWM apparatus was the same as in Experiment 1, except that the balls that were launched were not identical to the balls that were thrown, because we thought this might confound the perceptual size judgments of the launched object. Thus, the ball that was thrown was identical to that thrown in Experiment 1 , but the launched balls that the children were instructed to catch were regular tennis balls $(6.75 \mathrm{~cm}$ in diameter). In addition, in order to prevent the children from gathering haptic information about ball size, we had the children use a scoop net (opening $=$ $28 \mathrm{~cm}$ in diameter) to catch the ball, and the experimenter removed the ball from the net after it was caught. For the perception tests, we used nine black circles, each printed on a separate A4-size sheet of white paper. The circles measured between 5.75 and $7.75 \mathrm{~cm}$ in diameter, in increments of $0.25 \mathrm{~cm}$.

Procedure. Children were tested individually. They were instructed to catch the tennis ball with the scooping net after it was launched, and were told that they could launch the tennis ball by throwing the softball at the circle-shaped target. They did not receive instructions about how to catch the ball (e.g., whether to use the left or right hand for catching). By pushing against the target with his hand, the experimenter shortly demonstrated how the SWM launched the second ball. The child was encouraged to catch this ball with the net.

After 15 attempts, the children were taken to the other end of the gym, where the circles were arranged on the floor in a random fashion (the positions of the circles were randomly distributed for each child). The children were instructed to look carefully and pick the circle they thought best matched the size of the ball that was launched (and that they were told to catch with the net). The children were allowed to look at the ball, which was located next to the SWM, while performing the perception tests. All children received a chocolate-marshmallow for participation.

Data analysis. The number of balls that hit the target (out of 15 attempts) and the number of caught balls served as the performance measures. The perception measure was the diameter of the circle that the child indicated as matching the size of the tennis ball. Spearman rho correlations were performed to examine the strength of the relation between the number of caught balls or the number of target hits and the perceived ball size.

\section{Results and Discussion}

The catching task proved to be rather difficult for the children; less than half of the balls that were launched
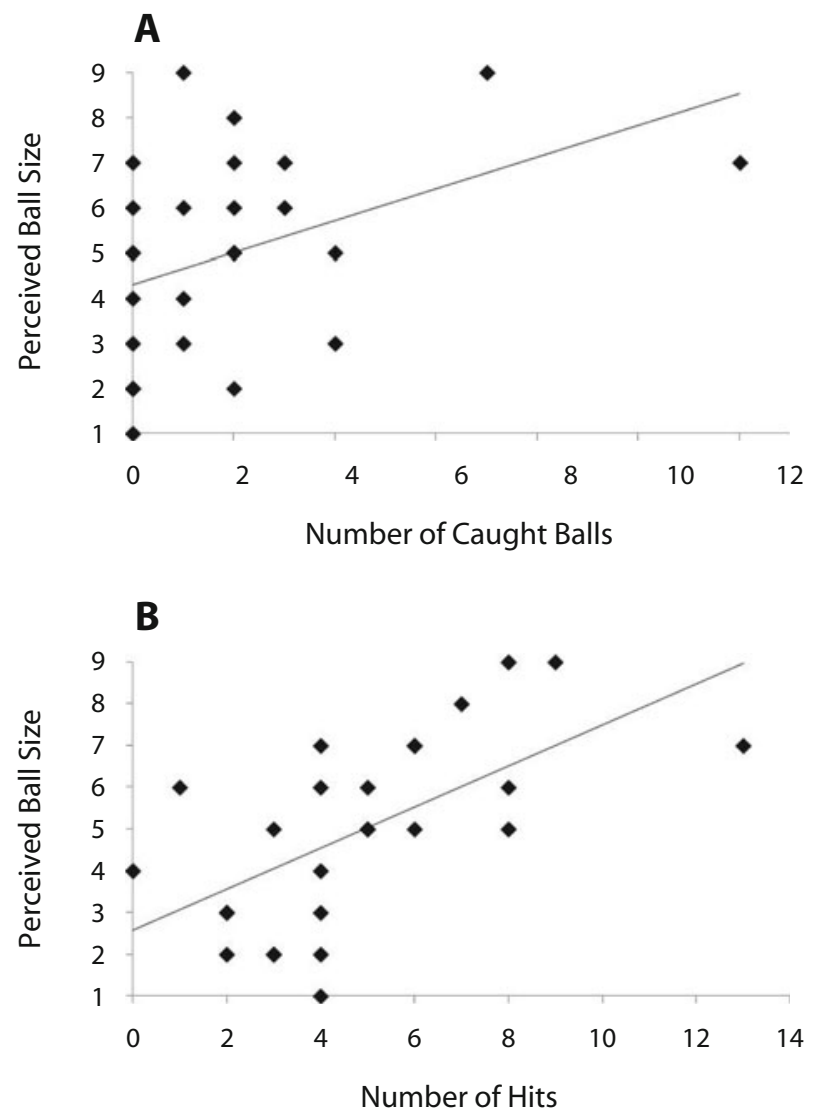

Figure 3. The relation between the number of caught balls (panel A) and the number of hits (panel B) and perceived ball size, ranging systematically from 1 (smallest) to 9 (biggest) in the throwing-and-catching task of Experiment 2. Each dot represents 1 or more participants. 
were actually caught (i.e., 49 out of 126; see Table 1). Yet, as predicted by the perceptual accentuation hypothesis, the results revealed a significant positive relation between the number of caught balls and the size judgments of the tennis ball $(r=.459, p<.001)$ (Figure 3A). In other words, action-specific influences on the perception of the end goal object occurred: Children who intercepted the ball more often with the net judged the ball to be bigger than children who were less successful. This indicates that the disappearance of an action-specific influence on perception of the intermediate goal object (i.e., the throwing target) in Experiment 1 cannot be explained by incomplete forward planning of the sequential task, by task complexity, or by visual properties simply vanishing from memory due to the additional time needed to complete the throwing-and-catching condition (as compared with the time needed to complete the throwing-only condition).

Interestingly, however, the relation between the number of balls that hit the target and the perceived size of the launched ball was also significant $(r=.665, p<.001)$ (Figure 3B). This may suggest that, in keeping with the perceptual accentuation hypothesis, the mere intention to catch the ball was sufficient-or even more important than actually catching it - for the emergence of actionspecific influences on perception.

\section{GENERAL DISCUSSION}

Recent evidence has demonstrated action-specific influences on perception of the environment (Wesp et al., 2004; Witt et al., 2008; Witt \& Proffitt, 2005, 2008). Successful performance systematically affects the perceived size or distance of action-related objects. Yet, despite a growing body of empirical evidence, the processes that mediate the occurrence of these action-specific influences remain largely unknown.

The present experiments show that the intended goal of an action is an important determinant for the emergence of action-specific influence on perception (see also Bekkering \& Neggers, 2002; Vishton et al., 2007; Witt, Proffitt, \& Epstein, 2004, 2005). The influence, however, is limited to objects that are related to the end goal of an action and do not encompass objects related to intermediate goals. Thus, when the children were instructed only to throw the ball at the circle-shaped target, making the target the end goal object, the perceived target size was influenced (Experiment 1). Similarly, when the children were told to catch the launched object, making the ball the end goal object, it was the perceived ball size that was mediated by performance level (Experiment 2). However, the latter instruction also turned the circle-shaped target into an intermediate goal object that was subordinate to the primary goal of intercepting the launched ball. Consequently, the action-specific effect on perceived target size that was apparent in the throwing-only condition vanished (Experiment 1).

Taken together, these findings provide strong support for the perceptual accentuation hypothesis (Bruner, 1957; Veltkamp et al., 2008). Perceptual accentuation (i.e., that objects are perceived as being bigger or nearer than they are) occurs only for objects that are the primary objects for action. Such accentuation of the end goal object is thought to make it easier to perceptually distinguish the object from other nearby objects that are less relevant to one's action intentions. We show that this perceptual accentuation is dependent on performance level. In accordance with the perceptual accentuation hypothesis, the present findings also suggest that the intention or motivation to act upon an object is sufficient to elicit the action-specific influences on perception (Veltkamp et al., 2008; Witt \& Proffitt, 2008). That is, in the catching task, a significant relation also emerged between hitting performance (i.e., actively launching the ball to be caught) and perceived ball size, suggesting that the action-specific influences did occur, independent of whether the ball was actually caught.

Recently, Witt and Proffitt (2008) proposed another account for the occurrence of action-specific influences on perception. They proposed that actors internally simulate, either covertly or explicitly, the execution of an intended action (see e.g., Jeannerod, 1999, 2001), and that it is the outcomes of these motor simulations that affect the perception of action-relevant objects (see also Hommel, Müsseler, Aschersleben, \& Prinz, 2001; Prinz, 1997). Consistent with this proposal, they showed that action-specific influences on perception are eliminated when observers perform a concurrent task that engages motor simulation processes. Clearly, the relative merits of the perceptual accentuation and the motor simulation accounts need further scrutiny. In this respect, it is important to notice that, whereas these accounts may provide an explanation for the emergence of action-specific influences on perception, both may have difficulty explaining why the influence on perception differs as a function of performance efficacy. Visual attention is a possible candidate. Ample evidence exists from visual gaze recordings that the spatiotemporal characteristics of visual information pickup differ as a function of the actor's skill and performance level (e.g., Mann, Williams, Ward, \& Janelle, 2007). For example, it has been suggested that skilled performance is associated with longer gaze fixation on task-relevant objects, such as the ball in baseball (Bahill \& LaRitz, 1984) or the ball and hole in golf (Vickers, 1992).

\section{AUTHOR NOTE}

We are grateful to our colleague Marco Hoozemans for statistical guidance with the regression analysis. We thank the teachers, parents, and children of the Kindergarten "Erlengrund" for their collaboration. Special thanks for their assistance with the experimental setup and procedure go to Cornelia, Andres, Tom, and Alistair. Correspondence concerning this article should be addressed to R. Cañal-Bruland, Research Institute MOVE, Faculty of Human Movement Sciences, VU University Amsterdam, Van der Boechorststraat 9, 1081 BT Amsterdam, The Netherlands (e-mail: r.bruland@fbw.vu.nl).

\section{REFERENCES}

Bahill, A. T., \& LaRitz, T. (1984). Why can't batters keep their eyes on the ball? American Scientist, 72, 249-253.

BeKKering, H., \& NegGers, S. F. W. (2002). Visual search is modulated by action intentions. Psychological Science, 13, 370-374.

Bruner, J. S. (1957). On perceptual readiness. Psychological Review, 64, 123-152. 
Bruner, J. S., \& Postman, L. (1949). Perception, cognition, and behavior. Journal of Personality, 18, 14-31.

Hommel, B., Müsseler, J., Aschersleben, G., \& Prinz, W. (2001). The theory of event coding (TEC): A framework for perception and action planning. Behavioral \& Brain Sciences, 24, 849-937.

JeAnNERoD, M. (1999). The 25th Bartlett Lecture: To act or not to act: Perspectives on the representation of actions. Quarterly Journal of Experimental Psychology, 52A, 1-29.

JEANNEROD, M. (2001). Neural simulation of action: A unifying mechanism for motor cognition. NeuroImage, 14, S103-S109.

Klein, G. S., Schlesinger, H. J., \& Meister, D. E. (1951). The effect of personal values on perception: An experimental critique. Psychological Review, 58, 96-112.

LIANG, K.-Y., \& ZEGER, S. L. (1993). Regression analysis for correlated data. Annual Review of Public Health, 14, 43-68.

Mann, D. T. Y., Williams, A. M., Ward, P., \& Janelle, C. M. (2007). Perceptual-cognitive expertise in sport: A meta-analysis. Journal of Sport \& Exercise Psychology, 29, 457-478.

PRINZ, W. (1997). Perception and action planning. European Journal of Cognitive Psychology, 9, 129-154.

ProffitT, D. R. (2006). Embodied perception and the economy of action. Perspectives on Psychological Science, 1, 110-122.

VeltKamp, M., AarTs, H., \& CuSTERs, R. (2008). Perception in the service of goal pursuit: Motivation to attain goals enhances the perceived size of goal-instrumental objects. Social Cognition, 26, 720-736

Vickers, J. N. (1992). Gaze control in putting. Perception, 21, 117 132.

Vishton, P. M., Stephens, N. J., Nelson, L. A., Morra, S. E., BruNICK, K. L., \& Stevens, J. A. (2007). Planning to reach for an object changes how the reacher perceives it. Psychological Science, 18, 713719 .

Wesp, R., Cichello, P., Gracia, E. B., \& Davis, K. (2004). Observing and engaging in purposeful actions with objects influences estimates of their size. Perception \& Psychophysics, 66, 1261-1267.

Witt, J. K., Linkenauger, S. A., Bakdash, J. Z., \& Proffitt, D. R. (2008). Putting to a bigger hole: Golf performance relates to perceived size. Psychonomic Bulletin \& Review, 15, 581-585.

WitT, J. K., \& ProffitT, D. R. (2005). See the ball, hit the ball: Apparent ball size is correlated with batting average. Psychological Science, 16, 937-938.

WitT, J. K., \& Proffitt, D. R. (2008). Action-specific influences on distance perception: A role for motor simulation. Journal of Experimental Psychology: Human Perception \& Performance, 34, 14791492.

Witt, J. K., Proffitt, D. R., \& Epstein, W. (2004). Perceiving distance: A role of effort and intent. Perception, 33, 570-590.

Witt, J. K., Proffitt, D. R., \& Epstein, W. (2005). Tool use affects perceived distance, but only when you intend to use it. Journal of Experimental Psychology: Human Perception \& Performance, 31, 880-888.

\section{NOTE}

1. Note that there was no significant relation between the number of caught balls and the target size perception either $(r=-.152, p>.10)$.

(Manuscript received April 16, 2009; revision accepted for publication August 25, 2009.) 